\title{
Microwave radio emissions as a proxy for coronal mass ejection speed in arrival predictions of interplanetary coronal mass ejections at $1 \mathrm{AU}$
}

\author{
Carolina Salas Matamoros ${ }^{1,2, *}$, Karl Ludwig Klein ${ }^{1}$, and Gerard Trottet ${ }^{1}$ \\ 1 LESIA-UMR 8109 - Observatoire de Paris, PSL Res. Univ., CNRS, Univ. P \& M Curie and Paris-Diderot, \\ 92190 Meudon, France \\ 2 Space Research Center, University of Costa Rica, 2060 San Jose, Costa Rica \\ *Corresponding author: carolina.salas@obspm.fr
}

Received 27 May 2016 / Accepted 26 October 2016

\begin{abstract}
The propagation of a coronal mass ejection (CME) to the Earth takes between about $15 \mathrm{~h}$ and several days. We explore whether observations of non-thermal microwave bursts, produced by near-relativistic electons via the gyrosynchrotron process, can be used to predict travel times of interplanetary coronal mass ejections (ICMEs) from the Sun to the Earth. In a first step, a relationship is established between the CME speed measured by the Solar and Heliospheric Observatory/Large Angle and Spectrometric Coronagraph (SoHO/LASCO) near the solar limb and the fluence of the microwave burst. This relationship is then employed to estimate speeds in the corona of earthward-propagating CMEs. These speeds are fed into a simple empirical interplanetary acceleration model to predict the speed and arrival time of the ICMEs at Earth. The predictions are compared with observed arrival times and with the predictions based on other proxies, including soft X-rays (SXR) and coronographic measurements. We found that CME speeds estimated from microwaves and SXR predict the ICME arrival at the Earth with absolute errors of $11 \pm 7$ and $9 \pm 7 \mathrm{~h}$, respectively. A trend to underestimate the interplanetary travel times of ICMEs was noted for both techniques. This is consistent with the fact that in most cases of our test sample, ICMEs are detected on their flanks. Although this preliminary validation was carried out on a rather small sample of events (11), we conclude that microwave proxies can provide early estimates of ICME arrivals and ICME speeds in the interplanetary space. This method is limited by the fact that not all CMEs are accompanied by non-thermal microwave bursts. But its usefulness is enhanced by the relatively simple observational setup and the observation from ground, which makes the instrumentation less vulnerable to space weather hazards.
\end{abstract}

Key words. Coronal mass ejections - Interplanetary coronal mass ejections - Flares - Radio bursts

\section{Introduction}

Coronal Mass Ejections (CMEs) are a major space weather hazard because they disturb the Earth's magnetosphere and may induce strong electric currents in the ionosphere and in the crust (e.g. Zhang et al. 2007). For this reason, one of the principal aims of space weather forecasting is the prediction of the travel time of these magnetic structures from the Sun to the Earth. CMEs take between about $15 \mathrm{~h}$ (Cliver et al. 1990) and a few days to reach the Earth. Prediction techniques have been developed based on remote observations and validated by in situ measurements. Most of these techniques involve two essential elements: the radial propagation speed of the CME in the corona and the interplanetary acceleration or deceleration.

CME velocities are usually obtained from coronographic observations of the time-height evolution of the front of the CME projected in the plane of the sky. But when the CME travels earthward, only the expansion speed perpendicular to the direction of propagation can be measured from the Sun-Earth line. Combined coronographic observations by the Solar Terrestrial Relations Observatory (STEREO) and Solar and Heliospheric Observatory (SoHO) spacecraft were a great step forward by enabling observations of the Sun with three eyes in different positions. 3D reconstruction techniques describe the propagation of CMEs in the corona (Thernisien et al. 2009). The combination with heliospheric imaging makes it possible to track CMEs from the Sun to the Earth (e.g. Rouillard 2011; Colaninno et al. 2013; Möstl et al. 2014) and, as a consequence, to develop and validate new prediction techniques (e.g. Gopalswamy et al. 2013).

Different models have been developed to describe the interplanetary propagation of ICMEs. Empirical models of interplanetary propagation are based on relationships between coronographic measurements and ICME parameters in the interplanetary space (e.g. Gopalswamy et al. 2001; Schwenn et al. 2005). More sophisticated techniques include MHD modelling of ICME propagation in the heliosphere. Models such as H3DMHD (Wu et al. 2011) and WSA-ENLIL+Cone (Odstrcil et al. 2004) use the coronal and in situ observations as input to simulate the propagation of disturbances into the heliosphere and predict their arrivals. Kinematic methods based on MHD or HD models have also been developed analytically (Cargill 2004; Vršnak \& Žic 2007).

The unique capabilities of the combined STEREO and SoHO missions will only exist for a limited time. Space weather monitoring, which relies on the continuous availability of data, will need alternative methods to estimate the propagation speed of CMEs. X-ray and radio emissions might serve this purpose. A direct empirical relationship was 
established between a proxy of the $3 \mathrm{GHz}$ fluence and the travel time of interplanetary shock waves between the Sun and the Earth by Caroubalos (1964), well before the actual discovery of CMEs themselves. Tobiska et al. (2013) developed a similar technique using soft X-ray fluence. The present study follows a different line of reasoning. It investigates whether gyrosynchrotron emission from non-thermal electrons at $\mathrm{GHz}$ frequencies (microwaves) can be used to infer the speed of Earth-directed CMEs in the corona, which can then be fed into an interplanetary acceleration model. The approach is identical to the work by Salas-Matamoros \& Klein (2015), which uses soft X-rays (SXR). In Section 2, empirical relationships are established between the speed of limb-CMEs and microwave fluence. In Section 3, we use these relationships in a sample containing 11 Earth-directed CMEs to estimate their outward propagation speeds without resorting to coronographic measurements. These speeds, together with a simple analytical formula for interplanetary acceleration or deceleration, are used to estimate the arrival times and speeds of the interplanetary coronal mass ejections (ICMEs) near 1 AU. The predictions are compared with in situ observations to evaluate their quality. The possible influence of the geometry of the ICME on the estimation of arrival time is explored. The results are discussed with respect to other efforts for arrival time prediction in Section 4.

\section{An empirical relationship between CME speed and microwave emission in limb events}

\subsection{Selection of the events}

The data set that we use in this study is based on the 49 events listed in Table 1 in Salas-Matamoros \& Klein (2015). Additionally, we incorporated events that occurred between 2009 and 2014. These CME events (with speeds of more than $100 \mathrm{~km} \mathrm{~s}^{-1}$ ) were carefully selected for their association with flares near the limb, at a central meridian distance between $70^{\circ}$ and $85^{\circ}$, and with soft X-ray (SXR) bursts. The SXR burst must be unambiguous: cases where bursts from different active regions could be associated with one given $\mathrm{CME}$, and cases where the SXR burst occurred far away from the CME were discarded. For this sample, we analysed the microwave data provided by the US Air Force Radio Solar Telescope Network $(\mathrm{RSTN})^{1}$ at $1.41,2.69,4.99,8.80$ and $15.40 \mathrm{GHz}$ and by the Nobeyama Radio Polarimeters (NoRP) $)^{2}$ at 1, 2, 3.75, 9.40, 17 and $35 \mathrm{GHz}$ (Nakajima et al. 1985). We searched for microwave emission that can be attributed to the gyrosynchrotron mechanism of mildly relativistic electrons (energy range $\sim 100 \mathrm{keV}$-several $\mathrm{MeV}$ ).

Whenever possible, microwave time profiles observed by different instruments were compared, and time profiles without data gaps were selected. We discarded 19 events where the data were not available or incomplete, 7 events where no microwave emission was seen in relationship with the SXR burst (Fig. 1a) and the event on 16 June 2004, where the smooth profile with time scales similar to the one observed for SXR emission was identified as thermal emission (Fig. 1b). The final CME/microwave burst sample contains 41 events, listed in Table 1.

\footnotetext{
$\overline{1} \mathrm{ftp} / / \mathrm{ftp} . n g d c . n o a a . g o v / \mathrm{STP} /$ space-weather/solar-data/solar-features/ solar-radio/rstn-1-second/

${ }^{2} \mathrm{ftp} / / /$ solar-pub.nao.ac.jp/pub/nsro/norp/xdr/
}
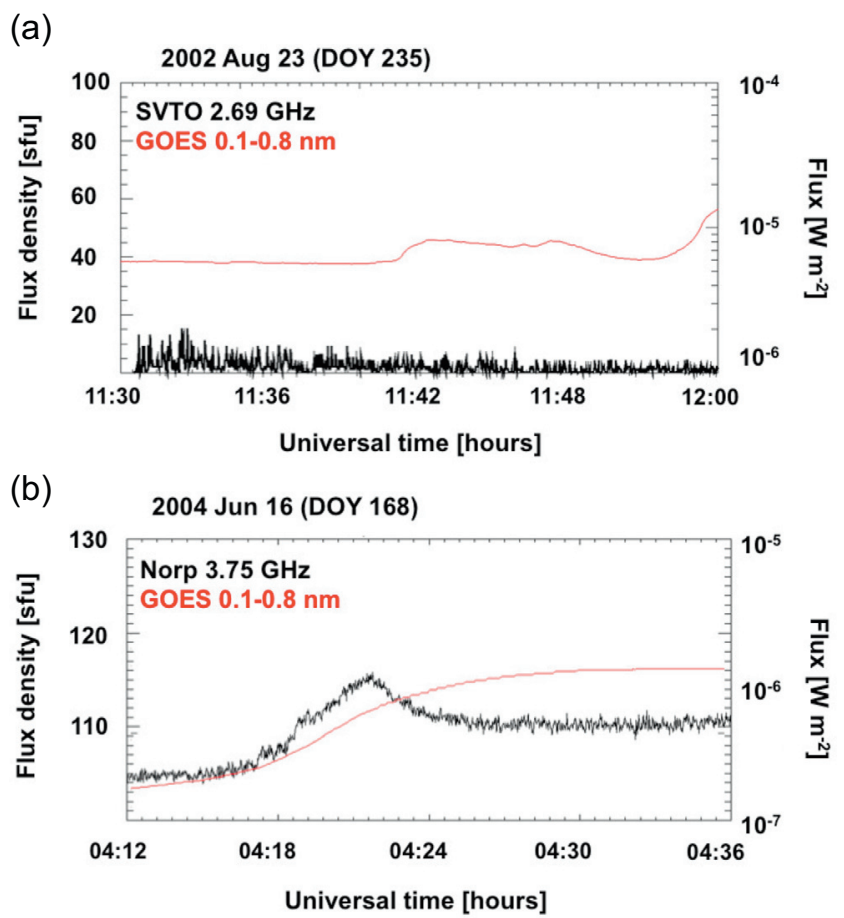

Fig. 1. Flux density profiles of discarded events. (a) No microwave emissions associated with the SXR burst. (b) Thermal microwave emissions.

(a)

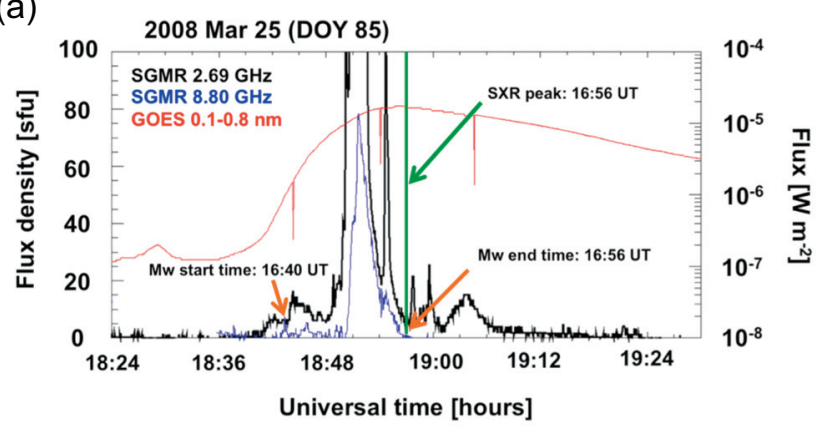

(b)

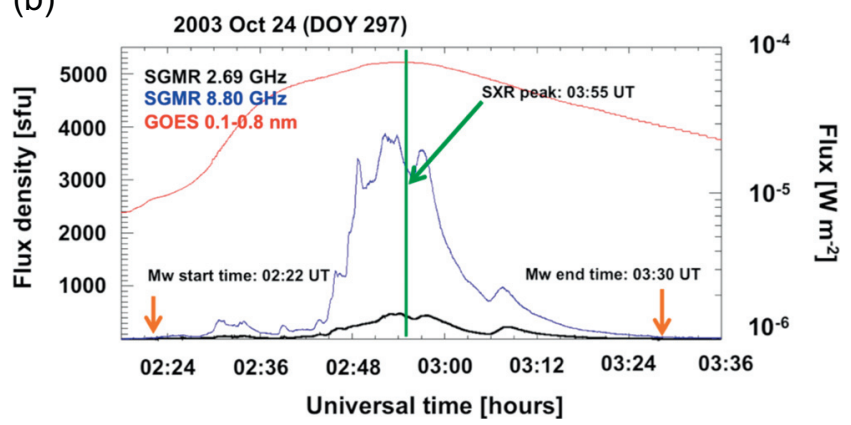

Fig. 2. Examples of time range selection for the fluence calculation. Black and blue curves are the microwave emissions at 2.69 and $8.8 \mathrm{GHz}$, respectively, while the SXR burst is plotted in red. The orange arrows show the start and the end of the time interval for the fluence calculation. (a) End of the interval matches the peak of the SXR counterpart (green line). (b) The microwave fluence calculation is extended until the end of the non-thermal microwave burst profile. 
Table 1. Table of events: event number (col. 1), date (col. 2), time of the first appearance of the CME in LASCO/C2 coronograph (col. 3 ), CME speed in the plane of the sky reported in LASCO-CME (col. 4), SXR peak time (col. 5), times of onset (col. 6), end (col. 7), fluences at $3 \mathrm{GHz}$ (col. 8), $9 \mathrm{GHz}$ (col. 9), maximum (col. 10) of microwave bursts. Lower limits of the maximum fluence mean that the real maximum was outside the observed frequency range.

\begin{tabular}{|c|c|c|c|c|c|c|c|c|c|}
\hline \multirow[t]{2}{*}{ No } & \multirow[t]{2}{*}{ Date } & \multicolumn{2}{|c|}{ CME parameters } & \multirow{2}{*}{$\frac{\mathrm{SXR}}{t_{\mathrm{p}}}$} & \multicolumn{5}{|c|}{ Microwave parameters } \\
\hline & & $t_{0}$ & $\begin{array}{c}V_{\mathrm{CME}} \\
{\left[\mathrm{km} \mathrm{s}^{-1}\right]}\end{array}$ & & $t_{0}$ & $t_{\mathrm{f}}$ & $\phi_{3 \mathrm{GHz}}[\mathrm{sfu} \cdot \mathrm{s}]$ & $\phi_{9 \mathrm{GHz}}[\mathrm{sfu} \cdot \mathrm{s}]$ & $\begin{array}{c}\phi_{\max } \\
{[\mathrm{sfu} \cdot \mathrm{s}]}\end{array}$ \\
\hline (1) & (2) & (3) & (4) & (5) & (6) & (7) & (8) & (9) & $(10)$ \\
\hline 1 & 19980425 & $15: 11$ & 349 & $14: 37$ & $15: 11$ & $14: 50$ & $2.3 \times 10^{4}$ & - & $\geq 3.1 \times 10^{4}$ \\
\hline 2 & 19990403 & $23: 47$ & 923 & $23: 10$ & $23: 00$ & $23: 17$ & $1.7 \times 10^{4}$ & $1.6 \times 10^{4}$ & $3.2 \times 10^{4}$ \\
\hline 3 & 19991108 & $07: 26$ & 154 & 06:01 & $05: 55$ & 06:01 & $\leq 1.4 \times 10^{2}$ & $\leq 2.8 \times 10^{2}$ & $\leq 3.6 \times 10^{2}$ \\
\hline 4 & 20000617 & $03: 28$ & 857 & $02: 37$ & $02: 30$ & $02: 40$ & $9.3 \times 10^{3}$ & $1.1 \times 10^{4}$ & $1.1 \times 10^{4}$ \\
\hline 5 & 20000623 & $14: 54$ & 847 & $14: 32$ & $14: 20$ & $14: 37$ & $4.8 \times 10^{4}$ & $2.2 \times 10^{5}$ & $2.2 \times 10^{5}$ \\
\hline 6 & 20010415 & $14: 06$ & 1199 & $13: 50$ & $13: 43$ & $13: 58$ & $4.0 \times 10^{5}$ & $5.3 \times 10^{5}$ & $>1.2 \times 10^{6}$ \\
\hline 7 & 20010810 & $02: 06$ & 376 & $01: 36$ & $01: 30$ & 01:39 & $3.9 \times 10^{3}$ & $3.8 \times 10^{3}$ & $\geq 7.2 \times 10^{3}$ \\
\hline 8 & 20011101 & $14: 30$ & 1053 & $15: 01$ & $14: 15$ & $16: 00$ & $6.5 \times 10^{5}$ & $4.8 \times 10^{5}$ & $6.5 \times 10^{5}$ \\
\hline 9 & 20011229 & $09: 54$ & 634 & $09: 45$ & $09: 39$ & $09: 45$ & $1.1 \times 10^{4}$ & $1.7 \times 10^{4}$ & $1.7 \times 10^{4}$ \\
\hline 10 & 20020313 & $23: 54$ & 489 & $23: 36$ & $23: 09$ & $23: 36$ & $1.7 \times 10^{4}$ & $2.0 \times 10^{4}$ & $2.5 \times 10^{4}$ \\
\hline 11 & 20020404 & $05: 06$ & 468 & $04: 40$ & $04: 25$ & $04: 42$ & $9.6 \times 10^{3}$ & $7.4 \times 10^{3}$ & $\geq 9.6 \times 10^{3}$ \\
\hline 12 & 20020705 & $13: 31$ & 818 & $13: 26$ & $13: 12$ & $13: 26$ & $2.2 \times 10^{4}$ & $1.5 \times 10^{4}$ & $\geq 2.2 \times 10^{4}$ \\
\hline 13 & 20020803 & $19: 31$ & 1150 & $19: 07$ & $19: 02$ & $19: 13$ & $1.3 \times 10^{4}$ & $3.0 \times 10^{4}$ & $\geq 3.0 \times 10^{4}$ \\
\hline 14 & 20020816 & $06: 06$ & 1378 & $06: 12$ & $05: 43$ & $07: 18$ & $1.1 \times 10^{5}$ & $3.4 \times 10^{5}$ & $\geq 1.1 \times 10^{7}$ \\
\hline 15 & 20020822 & $18: 26$ & 750 & $18: 02$ & $17: 35$ & 18:08 & $1.8 \times 10^{4}$ & $6.8 \times 10^{3}$ & $\geq 3.3 \times 10^{4}$ \\
\hline 16 & 20020908 & 02:06 & 364 & $01: 43$ & $01: 36$ & $\begin{array}{c}01: 45- \\
01: 53\end{array}$ & $(1.6-2.2) \times 10^{4}$ & $(2.9-4.7) \times 10^{4}$ & $\geq 2.9 \times 10^{4}$ \\
\hline 17 & 20030409 & $23: 50$ & 511 & $23: 29$ & $23: 26$ & $23: 33$ & - & $8.1 \times 10^{3}$ & $>8.1 \times 10^{3}$ \\
\hline 18 & 20030425 & $05: 50$ & 806 & $05: 40$ & $05: 22$ & $05: 58$ & $2.1 \times 10^{4}$ & $3.6 \times 10^{3}$ & $\geq 3.6 \times 10^{3}$ \\
\hline 19 & 20031023 & $20: 06$ & 1136 & $20: 03$ & $19: 52$ & $\begin{array}{c}20: 04- \\
20: 36\end{array}$ & $(2.7-9.8) \times 10^{4}$ & $7.3 \times 10^{4}-1.2 \times 10^{5}$ & $\geq 1.9 \times 10^{5}$ \\
\hline 20 & 20031024 & $02: 54$ & 1055 & $02: 55$ & $02: 22$ & $03: 30$ & $5.1 \times 10^{5}$ & $3.6 \times 10^{6}$ & $\geq 3.9 \times 10^{6}$ \\
\hline 21 & 20031103 & $10: 06$ & 1420 & $09: 56$ & $09: 48$ & $09: 57$ & $7.4 \times 10^{5}$ & $9.4 \times 10^{5}$ & $\geq 1.2 \times 10^{6}$ \\
\hline 22 & 20040107 & 04:06 & 1581 & 04:03 & $03: 50$ & $04: 19$ & $2.7 \times 10^{4}$ & $4.0 \times 10^{5}$ & $\geq 5.7 \times 10^{5}$ \\
\hline 23 & 20040107 & $10: 30$ & 1822 & $10: 26$ & $10: 15$ & $10: 34$ & $1.9 \times 10^{5}$ & $2.6 \times 10^{5}$ & $3.1 \times 10^{5}$ \\
\hline 24 & 20040517 & $05: 26$ & 383 & $04: 17$ & $04: 13$ & $04: 19$ & $1.7 \times 10^{3}$ & $2.5 \times 10^{3}$ & $\geq 2.5 \times 10^{3}$ \\
\hline 25 & 20040831 & $05: 54$ & 311 & $05: 38$ & $05: 31$ & $05: 38$ & $5.9 \times 10^{3}$ & $5.1 \times 10^{3}$ & $5.9 \times 10^{3}$ \\
\hline 26 & 20050417 & $21: 26$ & 721 & $21: 07$ & $21: 00$ & $21: 10$ & $6.9 \times 10^{3}$ & $6.6 \times 10^{3}$ & $\geq 1.1 \times 10^{4}$ \\
\hline 27 & 20050506 & $03: 30$ & 1120 & $03: 13$ & $03: 07$ & $03: 19$ & $1.8 \times 10^{3}$ & $3.1 \times 10^{4}$ & $3.3 \times 10^{4}$ \\
\hline 28 & 20050506 & $11: 54$ & 1144 & $11: 28$ & $11: 21$ & $11: 28$ & $6.9 \times 10^{3}$ & $2.3 \times 10^{4}$ & $2.3 \times 10$ \\
\hline 29 & 20050825 & $04: 54$ & 1327 & $04: 40$ & $04: 35$ & $04: 50$ & $3.7 \times 10^{4}$ & $3.9 \times 10^{5}$ & $\geq 6.4 \times 10^{5}$ \\
\hline 30 & 20060429 & $16: 54$ & 491 & $16: 30$ & $16: 21$ & $16: 35$ & $1.4 \times 10^{4}$ & $3.5 \times 10^{3}$ & $1.4 \times 10^{4}$ \\
\hline 31 & 20060430 & 02:06 & 428 & $01: 57$ & $01: 39$ & $\begin{array}{c}01: 59- \\
02: 11\end{array}$ & $(2.6-3.6) \times 10^{4}$ & $(2.3-8.7) \times 10^{4}$ & $\geq 1.0 \times 10^{5}$ \\
\hline 32 & $\begin{array}{c}200803 \\
25\end{array}$ & $19: 31$ & 1103 & $18: 56$ & $18: 40$ & $18: 57$ & $3.9 \times 10^{4}$ & $1.1 \times 10^{4}$ & $3.9 \times 10^{4}$ \\
\hline 33 & 20100614 & $01: 31$ & 343 & $00: 51$ & $00: 47$ & $00: 51$ & $6.7 \times 10^{2}$ & $5.4 \times 10^{2}$ & $7.0 \times 10^{2}$ \\
\hline 34 & 20110809 & $14: 00$ & 428 & $13: 45$ & $13: 29$ & $13: 45$ & $\leq 1.6 \times 10^{3}$ & $\leq 5.2 \times 10^{2}$ & $\leq 5.2 \times 10^{2}$ \\
\hline 35 & 20111114 & $20: 24$ & 383 & $20: 13$ & $19: 42$ & $20: 13$ & $\leq 8.8 \times 10^{2}$ & $\leq 7.8 \times 10^{2}$ & $\leq 1.9 \times 10^{3}$ \\
\hline 36 & 20111116 & $02: 12$ & 456 & 03:01 & $02: 55$ & 03:01 & $\leq 1.8 \times 10^{2}$ & $\leq 4.6 \times 10^{2}$ & $\leq 2.0 \times 10^{3}$ \\
\hline 37 & 20120606 & $03: 12$ & 375 & $02: 19$ & $02: 12$ & $02: 19$ & $\leq 1.2 \times 10^{2}$ & $\leq 4.6 \times 10^{2}$ & $\leq 1.6 \times 10^{3}$ \\
\hline 38 & 20120708 & $10: 48$ & 662 & $09: 53$ & $09: 44$ & $09: 53$ & $\leq 6.2 \times 10^{2}$ & $\leq 3.5 \times 10^{2}$ & $\leq 6.2 \times 10^{2}$ \\
\hline 39 & 20130717 & $09: 48$ & 355 & $09: 16$ & $09: 12$ & $09: 16$ & $<2.8 \times 10^{2}$ & $<1.8 \times 10^{2}$ & $<2.8 \times 10^{2}$ \\
\hline 40 & 20140214 & $17: 24$ & 283 & $16: 52$ & $16: 33$ & $16: 47$ & $2.1 \times 10^{4}$ & $1.4 \times 10^{4}$ & $\geq 4.1 \times 10^{4}$ \\
\hline 41 & 20140712 & $14: 24$ & 479 & $14: 08$ & $14: 02$ & $14: 13$ & $7.5 \times 10^{1}$ & $2.1 \times 10^{3}$ & $2.7 \times 10^{3}$ \\
\hline
\end{tabular}

\subsection{Fluence calculation}

For all microwave bursts, a background flux density ${ }^{3}$ was determined as the average in a suitable time interval before the microwave burst, and was subtracted. The fluence $(\phi)$ was calculated by integrating the flux density during a userdefined time interval. Figure 2 shows two examples. The time interval for the fluence calculation depends on the event under consideration. In impulsive bursts, the microwave emission

\footnotetext{
$\overline{{ }^{3} \text { Flux density is given }}$ in solar flux units (sfu): $1 \mathrm{sfu}=$ $10^{-22} \mathrm{~W} \mathrm{~m}^{-2} \mathrm{~Hz}^{-1}$.
}

occurs mainly during the rise phase of the SXR burst in the GOES $0.1-0.8 \mathrm{~nm}$ channel, as illustrated by the flux density profile at $8.8 \mathrm{GHz}$ (blue curve) in Figure 2a. In these cases, the fluence was calculated until the end of the broadband microwave burst as observed at frequencies $>5 \mathrm{GHz}$. The same end time was taken at all frequencies. This caused parts of the low-frequency microwave emission to be cut off when it had no counterpart at higher frequencies, such as the $2.69 \mathrm{GHz}$ burst after 19:00 UT in Figure 2a (black curve). Fluence was also calculated until the end of the microwave burst when a weak level of emission persisted in the early decay phase of the SXR burst, as illustrated in Figure 2b. The fluences at 
3 and $9 \mathrm{GHz}$, and the maximum fluence observed, are listed in cols. 8-10 in Table 1. In three cases (events 16, 19 and 31), the microwave bursts presented a new rise after the SXR peak. The microwave fluences with and without the late emission were computed and we found substantial changes in the fluence calculation for these events. Nevertheless, since these are only three events, they do not affect the statistical relationships and their late emission was not taken into account in the following analysis where we consider that the CME acceleration is most pronounced during the rise phase of the SXR burst (e.g. Maričić et al. 2007; Bein et al. 2012).

We use the fluence at $3 \mathrm{GHz}\left(\phi_{3 \mathrm{GHz}}\right), 9 \mathrm{GHz}\left(\phi_{9 \mathrm{GHz}}\right)$ and the maximum fluence $\left(\phi_{\max }\right)$ when it could be identified. The synchrotron spectrum rises with increasing frequency in its optically thick part and decreases at higher frequencies, where the emission is optically thin. The spectral maximum was within the range of observed frequencies in only 13 cases. A lower limit of the maximum fluence is listed in the last column of Table 1 when the highest fluence is found at the highest observed frequency. In event 1 , the emission at $9 \mathrm{GHz}$ was of thermal origin. In event 17, the $3 \mathrm{GHz}$ emission had a different time profile than the higher frequencies, suggesting it was due to collective plasma emission. In both cases, the fluence is not listed in Table 1. In the seven cases (events 3, 34-39) where no microwave burst could be identified, upper limits of the fluence are given. The upper limits were calculated as the product of the background standard deviation of the flux density and the duration of the rise phase of the SXR burst.

\subsection{Relationships between CME speed and microwave fluence}

Figure 3 shows the scatter plots between the logarithm of the limb-CME propagation speed $\left(V_{\mathrm{CME}}\right)$ and the logarithm of the fluence of the associated microwave bursts produced by gyrosynchrotron emission. The red arrows mark the upper limits of the fluence in the seven events where no microwave burst was seen. Lower limits of $\phi_{\max }$ are shown by blue arrows in Figure 3c. Even though some scatter remains in Figure 3 a clear relationship between CME speed and microwave fluence is obtained. Ignoring the upper and lower limits, we found significant correlations in all three cases:

- at $3 \mathrm{GHz}: r=0.56 \pm 0.14$ for 33 events and $p=0.07 \%$,

- at $9 \mathrm{GHz}: r=0.72 \pm 0.08$ for 33 events and $p=0.0002 \%$,

- maximum fluence: $r=0.75 \pm 0.15$ for 13 events and $p=0.3 \%$,

where $p$ is the probability of obtaining this or a higher correlation coefficient from a random sample of uncorrelated events. The errors were calculated using a bootstrap method, where the correlation coefficient was calculated repeatedly a 1000 times for a randomly selected sample of 33 or 13 from the observed data pairs, and the mean and standard deviation are quoted as the correlation coefficient and its statistical uncertainty. The correlations are slightly higher at $9 \mathrm{GHz}$ than at $3 \mathrm{GHz}$. That is expected, since the $3 \mathrm{GHz}$ gyrosynchrotron emission is optically thick, and hence only weakly related to the energy released to non-thermal electrons.

To find the statistical relationships between $V_{\mathrm{CME}}$ and $\phi$, we use three different linear fit methods to determine a straight line of the form

$$
\log V_{\mathrm{CME}}=A+B \log \phi,
$$
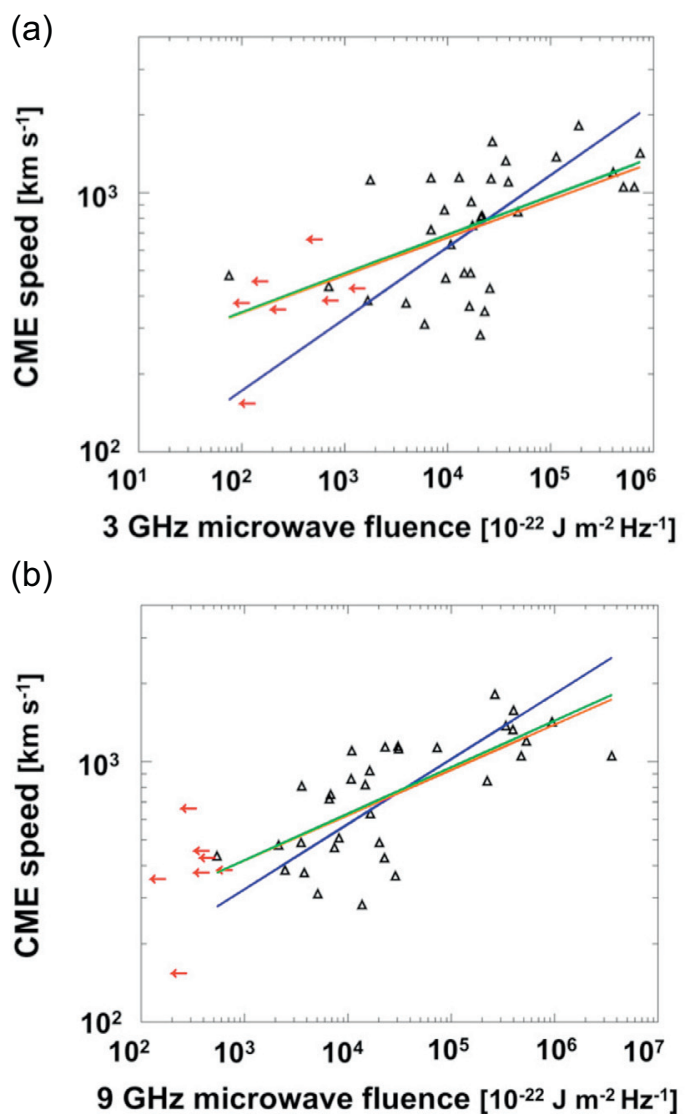

(c)

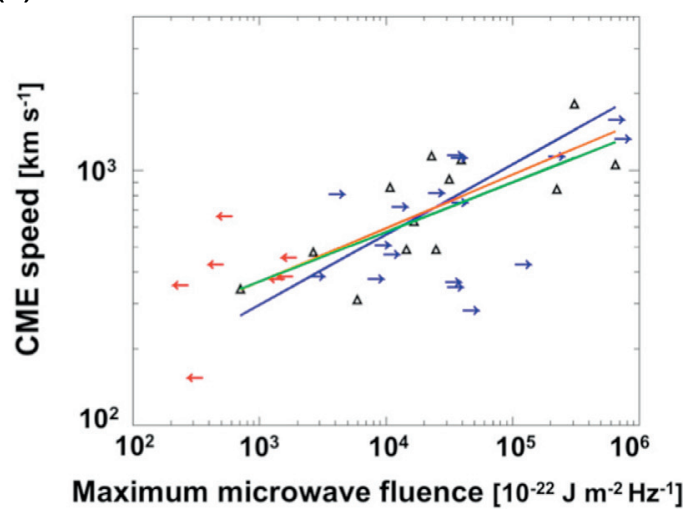

Fig. 3. Scatter plots for limb-CME speed over the period 1998-2014 versus the microwave fluences: (a) $\phi_{3 \mathrm{GHz}}$, (b) $\phi_{9 \mathrm{GHz}}$ and (c) $\phi_{\max }$. The solid coloured lines represent the linear fits obtained by different methods: least absolute deviation (green), least squares (orange) and total least squares (blue). Red arrows show upper limits of the fluences, blue arrows the lower limits of $\phi_{\max }$.

namely least squares (LS), least absolute deviation (LAD) and total least squares (TLS). Figure 2 shows the regression lines for the different methods. Table 2 contains the linear fit parameters of equation (1). The plots show that the methods of least absolute deviation (green line) and least squares (orange line) visually describe the sample well, including those observations where only upper limits were available. The TLS method (blue line), although it is formally more satisfactory since it assumes that both variables have uncertainties, provides a less convincing result, judging from visual inspection, when one includes the upper and lower 
C.S. Matamoros et al.: Microwave proxy in ICME arrival predictions

Table 2. Parameters of equation (1) using three different methods of linear fit.

\begin{tabular}{cccc}
\hline \hline Frequency & \multicolumn{3}{c}{ Linear fit method } \\
\cline { 2 - 4 } & Least squares & Least absolute deviation & Total least squares \\
\hline $3 \mathrm{GHz}$ & $A=2.25 \pm 0.29$ & $A=2.24 \pm 0.29$ & $A=1.68 \pm 0.41$ \\
& $B=0.14 \pm 0.06$ & $B=0.15 \pm 0.06$ & $B=0.28 \pm 0.10$ \\
$9 \mathrm{GHz}$ & $A=2.10 \pm 0.23$ & $A=2.09 \pm 0.23$ & $A=1.76 \pm 0.36$ \\
& $B=0.17 \pm 0.05$ & $B=0.18 \pm 0.05$ & $B=0.25 \pm 0.07$ \\
Maximum & $A=1.93 \pm 0.70$ & $A=1.98 \pm 0.70$ & $A=1.65 \pm 0.57$ \\
& $B=0.20 \pm 0.15$ & $B=0.19 \pm 0.15$ & $B=0.28 \pm 0.13$ \\
\hline
\end{tabular}

limits of the microwave fluence: the straight line appears too steep, leading to an overestimation of CME speeds for high microwave fluences, and an underestimation for low fluences. The LS and LAD methods provide comparable results when the statistical uncertainties are considered: the CME speeds calculated with the two methods at the extreme values of $9 \mathrm{GHz}$ fluence differ by less than the statistical uncertainty calculated with the values of Table 2 .

In the Section 3, we apply the correlations between $V_{\mathrm{CME}}$ and $\phi_{9 \mathrm{GHz}}$ obtained by the LS method to infer the speed of Earthward-directed CMEs, leaving aside $\phi_{3 \mathrm{GHz}}$, because it is optically thick. While the coefficients $A$ and $B$ in Table 2 are comparable, the dispersion is higher at $3 \mathrm{GHz}$. We did not use $\phi_{\max }$ either, because it could only be determined in few events.

\section{Application to ICME arrival time predictions}

\subsection{Event selection, fluence calculation, empirical identification of ICME arrival}

We collected a sample of Earth-directed CME events from the literature, which were related with flares located between $\pm 40^{\circ}$ from central meridian. We started with 26 events listed in Table 2 in Salas-Matamoros \& Klein (2015), which were used in this publication to infer the speed of CMEs from SXR emission. We discarded events where microwave data were either unavailable or of poor quality (events on 12 May 1997, 21 June 1998, 13 April 1999, 5 September 2002, 3 April 2010, 6 September 2011, 19 January 2012 and 10 March 2012), or where no microwave emission was associated with the SXR burst (events on 20 June 2000 and 28 July 2000). The 12 July 2012 event was also discarded because we could not affirm from the microwave profile at $9 \mathrm{GHz}$ that this was gyrosynchrotron emission. We selected 12 more ICME events to increase our sample: six (between 2011 and 2012) from the ISEST catalogue ${ }^{4}$, one from Table 1 in Shi et al. (2015) and five (in the years 2013 and 2014) from Table 1 in Mays et al. (2015).

In addition, the 20 events listed in Table 1 in Gopalswamy et al. (2013) were also considered. Eight events in this list were already in our original sample. Other events were discarded because of the location of the source (one event), the absence of clear non-thermal microwave emission (three events), the unavailability of radio observations (one event) or because more than one CME was observed with few hours difference.

For the total compiled sample of 26 CME-ICME pairs, we compared the arrival times found in the literature with the data

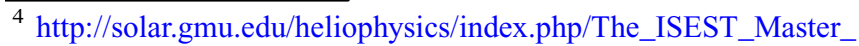
CME_List
}

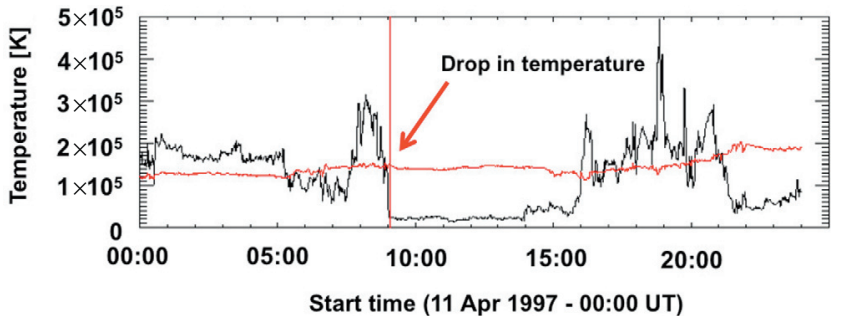

Fig. 4. Temperature profiles around and during the passage of an ICME at the Wind spacecraft. The black curve is the measured proton temperature. The red profile is the expected temperature (Eq. 2) for the observed solar wind speed as a function of time. The red arrow marks the ICME arrival time.

obtained by the Wind spacecraft. Gosling et al. (1973) found that ICME plasma is characterised by low proton temperature due to the expansion of the magnetic structure. Then, we can expect a drop in the observed proton temperature, when the ICME arrives at the spacecraft, below the expected temperature $\left(T_{\text {exp }}\right)$ of the standard solar wind at the same speed, given by Elliott et al. (2005) as

$$
T_{\exp }[\mathrm{K}]=640 \cdot V\left[\mathrm{~km} \mathrm{~s}^{-1}\right]-1.56 \times 10^{5}[\mathrm{~K}]
$$

For each ICME, we overplotted $T_{\exp }$ on the observed temperature profile as is shown in Figure 4 and searched for a decrease in the proton temperature near the ICME arrival time reported in the literature. In most events, the difference between the arrival time in the literature and the value determined using the drop in the proton temperature is less than $3 \mathrm{~h}$. In two events, the difference was larger: the ICME on 23 June 2013 was reported to arrive at 03:51 UT, but the very clear drop in temperature occurred at 16:13 UT. The strongest discrepancy was found for the ICME on 12 Mars 2012 (arrival reported at 08:30 UT but with a clear drop in temperature at 21:44 UT).

ICMEs with ambiguous observed arrival times, including events with multiple drops in the temperature that could not be uniquely related to the ICME arrival time, were discarded. The final sample contains 11 well-identified CME-ICME pairs whose origins were associated with non-thermal microwave emission. We calculated the $9 \mathrm{GHz}$ fluence as in the previous section, and determined the CME speed using equation (1) with the coefficients of Table 2 obtained through least squares minimisation. For comparison, we also calculated the CME speeds estimated from coronographic observations and from the SXR fluence, as described in Salas-Matamoros \& Klein (2015). 


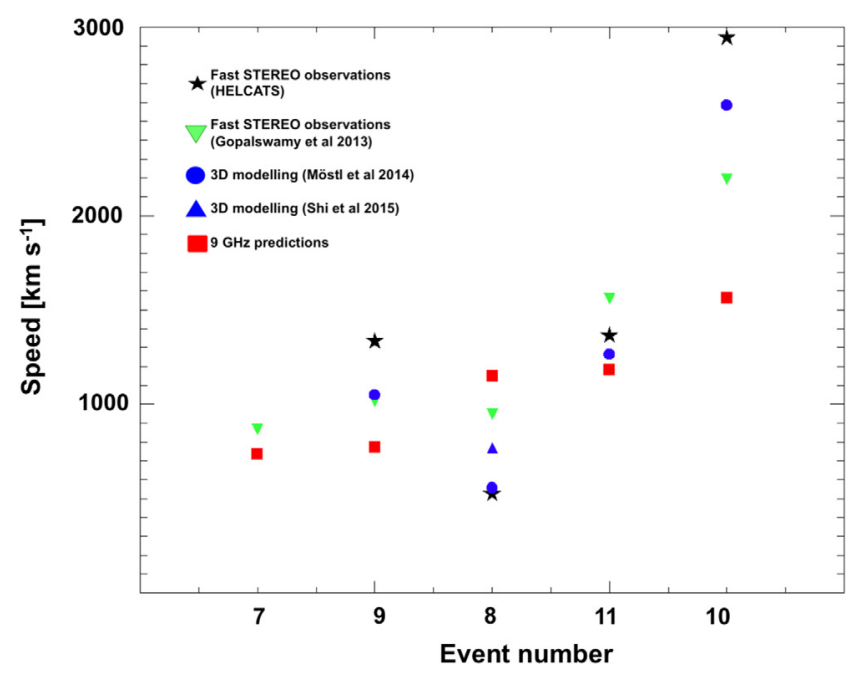

Fig. 5. Observed and predicted CME speeds. The black stars represent the higher of the two CME speeds derived from a geometric fit of observations from each of the two STEREO spacecraft (HELCATS catalogue). The green inverted triangles show the higher of the two CME speeds measured in the STEREO/ COR2 images by Gopalswamy et al. (2013). The blue circles and blue triangles are the speeds resulting from multi-spacecraft modelling by Möstl et al. (2014) and Shi et al. (2015). The red filled squares denote the speeds found from the microwave fluence by using least squares (LS) fitting.

\subsection{CME speed determination}

Before discussing the propagation of the 11 ICMEs to the Earth, we checked the CME speed estimate from the microwave fluence with coronographic measurements using STEREO and SoHO. The five events where such observations are available are plotted in Figure 5. The events are ordered by increasing predicted CME speed using the microwave fluence (red squares). The black stars represent the CME speeds found through a single spacecraft geometric fitting technique used in the HELCATS catalogue. ${ }^{5}$ This catalogue presents the fits to the observations of both STEREO spacecraft. Only the higher of the two speeds is plotted in Figure 5 because we assume that the higher speed is closer to the outward speed. The inverted green triangles are the speed measurements in that STEREO/COR2 FOV in which the CME was closest to the limb (Tab. 1 in Gopalswamy et al. 2013). The speeds resulting from multi-spacecraft modelling by Möstl et al. (2014) or Shi et al. (2015) are represented by filled blue circles and triangles, respectively.

The comparison between predictions, observations and modelling in Figure 5 reveals a large spread of CME speeds derived from different techniques. The predictions from the $9 \mathrm{GHz}$ fluence (filled red squares) tend to give lower $\mathrm{CME}$ speeds than the other estimations ( $4 / 5$ cases $)$. In the special case of event 8 , the predicted speed was found to be higher. However, the modelling of the speed at low altitudes $\left(\approx 2 \mathrm{R}_{\odot}\right)$ reveals a value of about $1100 \mathrm{~km} \mathrm{~s}^{-1}$ (Temmer et al. 2014), which is closer to the speed predicted from radiative proxies. This event also presented a CME-CME interaction studied by Temmer et al. (2014), which apparently affected the estimation of the speed. CME-CME interactions were also observed for events 9 and 10. CME interactions in

\footnotetext{
5 http://www.helcats-fp7.eu/catalogues/wp3_cat.html
}

the corona and in the interplanetary space can result in errors in both the speed estimations and the travel time predictions.

Overall Figure 5 shows that the discrepancy between microwave-inferred CME speeds and those derived from coronographic observations is not larger than the difference between different coronographic speed determinations themselves. Event 8 illustrates that even when 3D modelling is used, the speeds derived by different authors for the same event may differ substantially.

\subsection{Prediction of ICME arrival times}

CMEs undergo acceleration or deceleration in the corona (Vršnak et al. 2004) and interplanetary space (Gopalswamy et al. 2001). The latter was inferred from the observation that the range of ICME speeds near $1 \mathrm{AU}$ is smaller than the range of CME speeds in the corona (Gopalswamy et al. 2000). CME acceleration/deceleration is directly observed in heliospheric images from the STEREO mission (e.g. Colaninno et al. 2013).

Interplanetary propagation of ICMEs is generally discussed in terms of aerodynamic drag, i.e. a friction-like effect, between the outward propagating ICME and the ambient plasma (Vršnak et al. 2014 and references therein). Interplanetary deceleration is also expected when plasma is piled up in front of the ICME. Both processes are treated by the so-called "drag-based" model, where the force acting on the ICME is proportional to the square of the difference between the speeds of the ICME and the ambient solar wind, with a coefficient that may be determined empirically. Gopalswamy et al. (2001) and Gopalswamy (2009) proposed simple empirical relationships in first and second order of the velocity difference, and scaled them using observations of CMEs and the leading edge of ICMEs. The empirical laws are

$$
\begin{gathered}
a\left[\mathrm{~m} \mathrm{~s}^{-2}\right]=-0.0054\left(V_{\mathrm{CME}}-V_{01}\right), \\
a\left[\mathrm{~m} \mathrm{~s}^{-2}\right]=-3.29 \times 10^{-6}\left(V_{\mathrm{CME}}-V_{02}\right)^{2}-3.64 \\
\times 10^{-3}\left(V_{\mathrm{CME}}-V_{02}\right),
\end{gathered}
$$

where $V_{\mathrm{CME}}$ is the propagation speed of the CME in the corona, $V_{01}=406 \mathrm{~km} \mathrm{~s}^{-1}$ and $V_{02}=482 \mathrm{~km} \mathrm{~s}^{-1}$. These speeds have no specific physical meaning. In a model related to frictional drag, they could be considered as the equivalent ambient solar wind speed in the two acceleration models, respectively. We infer $V_{\mathrm{CME}}$ from the $9 \mathrm{GHz}$ fluence. The ICME acceleration or deceleration is assumed to occur within a maximum range out to $0.76 \mathrm{AU}$. It stops earlier if the ICME has reached the speed $V_{01}$ or $V_{02}$. With this event-dependent, but otherwise constant acceleration, the determination of the speed and arrival time of the ICME at $1 \mathrm{AU}$ is a matter of simple arithmetic. The results presented here were found using equation (3).

Table 3 summarises the ICME arrival time measurements and predictions. The event numbers are shown in col. 1. Cols. 2 and 3 contain, respectively, the shock arrivals and the arrival times of the magnetic obstacle identified by the temperature drop at the Wind spacecraft. Col. 4 presents the first time that the CME was observed by LASCO. Col. 5 lists the longitudes of the associated flares. Cols. 6 and 7 present the start and end times of microwave bursts, Col. 8 the $9 \mathrm{GHz}$ fluence. The predictions for the CME speed and arrival using microwave fluence are listed in Cols. 9 and 10, respectively. Cols. 11 and 12 contain the differences between the predicted 
Table 3. Comparison between ICME arrival times measured at Wind spacecraft and predicted based on $9 \mathrm{GHz}$ fluence: event number (col. 1), shock arrival (col. 2), ICME arrival (col. 3), CME onset (col. 4), flare longitude location (col. 5), microwave observations (cols. 6-8) and predictions based on the $9 \mathrm{GHz}$ fluence (cols. 9-12).

\begin{tabular}{|c|c|c|c|c|c|c|c|c|c|c|c|}
\hline \multirow[t]{2}{*}{ No } & \multirow{2}{*}{$\begin{array}{l}\text { Shock } \\
\text { onset } \\
\text { wind }\end{array}$} & \multirow{2}{*}{$\begin{array}{l}\text { ICME } \\
\text { onset } \\
\text { Wind }\end{array}$} & \multirow{2}{*}{$\begin{array}{l}\text { CME onset } \\
\text { LASCO }\end{array}$} & \multirow{2}{*}{$\begin{array}{c}\text { Flare } \\
\text { longitude }\end{array}$} & \multicolumn{3}{|c|}{ Microwave burst observations } & \multicolumn{4}{|c|}{$9 \mathrm{GHz}$ Predictions } \\
\hline & & & & & Start & End & $\begin{array}{r}\phi_{9 \mathrm{GHz}} \\
{[\mathrm{sfu} \cdot \mathrm{s}]}\end{array}$ & $\begin{array}{c}V_{\mathrm{CME}} \\
{\left[\mathrm{km} \mathrm{s}^{-1}\right]}\end{array}$ & Arrival & $\begin{array}{c}\Delta T_{\mathrm{ICME}} \\
{[\mathrm{h}]} \\
\end{array}$ & $\begin{array}{c}\Delta T_{\text {Sheath }} \\
{[\mathrm{h}]} \\
\end{array}$ \\
\hline (1) & $\begin{array}{c}(2) \\
1997\end{array}$ & (3) & (4) & (5) & (6) & (7) & $(8)$ & (9) & (10) & $(11)$ & $(12)$ \\
\hline 1 & $\begin{array}{c}\text { 11 Apr } \\
05: 00 \\
2000\end{array}$ & $\begin{array}{c}\text { 11 Apr } \\
\text { 09:07 }\end{array}$ & $\begin{array}{c}\text { 07 Apr } \\
14: 27\end{array}$ & $\mathrm{E} 11^{\circ}$ & $13: 54$ & $14: 20$ & $6.4 \times 10^{4}$ & 824 & $\begin{array}{c}10 \mathrm{Apr} \\
18: 05\end{array}$ & -15 & -11 \\
\hline $2 *$ & $\begin{array}{l}\text { 08 Jun } \\
09: 12\end{array}$ & $\begin{array}{c}08 \text { Jun } \\
13: 07\end{array}$ & $\begin{array}{c}\text { 06 Jun } \\
15: 54\end{array}$ & $\mathrm{E} 10^{\circ}$ & $15: 05$ & $16: 00$ & $2.0 \times 10^{6}$ & 1481 & $\begin{array}{c}\text { 08 Jun } \\
04: 05\end{array}$ & -9 & -5 \\
\hline 3 & $\begin{array}{l}28 \mathrm{Jul} \\
06: 00 \\
2003\end{array}$ & $\begin{array}{l}28 \mathrm{Jul} \\
13: 04\end{array}$ & $\begin{array}{l}25 \mathrm{Jul} \\
03: 30\end{array}$ & W08 & $02: 46$ & 02:51 & $1.5 \times 10^{5}$ & 950 & $\begin{array}{l}27 \mathrm{Jul} \\
19: 40\end{array}$ & -17 & -10 \\
\hline 4 & $\begin{array}{c}28 \text { Oct } \\
02: 06 \\
2005\end{array}$ & $\begin{array}{c}28 \text { Oct } \\
03: 20\end{array}$ & $\begin{array}{l}26 \text { Oct } \\
17: 54\end{array}$ & $\mathrm{~W} 38^{\circ}$ & $17: 20$ & $18: 14$ & $2.6 \times 10^{6}$ & 1554 & $\begin{array}{c}28 \text { Oct } \\
04: 10\end{array}$ & 1 & 2 \\
\hline 5 & $\begin{array}{c}15 \text { May } \\
02: 38 \\
2006\end{array}$ & $\begin{array}{c}15 \text { May } \\
09: 15\end{array}$ & $\begin{array}{c}\text { 13 May } \\
17: 12\end{array}$ & $\mathrm{E} 11^{\circ}$ & $16: 32$ & $17: 18$ & $5.5 \times 10^{5}$ & 1190 & $\begin{array}{c}15 \text { May } \\
17: 25\end{array}$ & 9 & 16 \\
\hline 6 & $\begin{array}{c}14 \mathrm{Dec} \\
14: 14 \\
2010\end{array}$ & $\begin{array}{c}14 \text { Dec } \\
20: 25\end{array}$ & $\begin{array}{c}13 \mathrm{Dec} \\
02: 54\end{array}$ & $\mathrm{~W} 23^{\circ}$ & $02: 18$ & 02:41 & $3.2 \times 10^{6}$ & 1607 & $\begin{array}{l}\text { 14 Dec } \\
11: 30\end{array}$ & -9 & -3 \\
\hline 7 & $\begin{array}{c}15 \text { Feb } \\
17: 28 \\
2011\end{array}$ & $\begin{array}{c}16 \text { Feb } \\
04: 53\end{array}$ & $\begin{array}{c}12 \mathrm{Feb} \\
13: 42\end{array}$ & $\mathrm{E} 11^{\circ}$ & $11: 22$ & $11: 38$ & $3.24 \times 10^{4}$ & 737 & $\begin{array}{c}15 \mathrm{Feb} \\
20: 58\end{array}$ & -8 & 3 \\
\hline 8 & $\begin{array}{c}18 \mathrm{Feb} \\
01: 30\end{array}$ & $\begin{array}{c}18 \mathrm{Feb} \\
04: 55\end{array}$ & $\begin{array}{c}15 \mathrm{Feb} \\
02: 24\end{array}$ & $\mathrm{~W} 10^{\circ}$ & $01: 48$ & $02: 14$ & $4.4 \times 10^{5}$ & 1148 & $\begin{array}{c}17 \mathrm{Feb} \\
05: 00\end{array}$ & -24 & -20 \\
\hline 9 & $\begin{array}{c}\text { 04 Aug } \\
22: 00 \\
2012\end{array}$ & $\begin{array}{c}05 \text { Aug } \\
03: 20\end{array}$ & $\begin{array}{c}\text { 02 Aug } \\
06: 39\end{array}$ & $\mathrm{~W} 10^{\circ}$ & $05: 48$ & $06: 23$ & $4.4 \times 10^{4}$ & 774 & $\begin{array}{l}5 \text { Aug } \\
13: 05\end{array}$ & 10 & 15 \\
\hline 10 & $\begin{array}{c}08 \mathrm{Mar} \\
11: 00\end{array}$ & $\begin{array}{c}09 \mathrm{Mar} \\
02: 40\end{array}$ & $\begin{array}{c}07 \mathrm{Mar} \\
00: 24\end{array}$ & $\mathrm{E} 26^{\circ}$ & $00: 10$ & 00:20 & $2.7 \times 10^{6}$ & 1562 & $\begin{array}{c}08 \mathrm{Mar} \\
10: 45\end{array}$ & -16 & 0 \\
\hline 11 & $\begin{array}{c}12 \mathrm{Mar} \\
08: 30\end{array}$ & $\begin{array}{l}12 \mathrm{Mar} \\
21: 44\end{array}$ & $\begin{array}{c}10 \mathrm{Mar} \\
18: 00\end{array}$ & $\mathrm{~W} 26^{\circ}$ & $17: 10$ & $18: 27$ & $5.2 \times 10^{5}$ & 1180 & $\begin{array}{c}12 \mathrm{Mar} \\
18: 30\end{array}$ & -3 & 10 \\
\hline
\end{tabular}

* The ICME arrival of event 2 was measured by ACE because no wind data were available.

arrival time and the arrival times of the ICME and the shock, respectively.

The procedure employed here, as well as in published studies, predicts the arrival time of the top (or nose) of the ICME at $1 \mathrm{AU}$. The CME propagation speed measured in the corona, which we used as input for the empirical relationship with the microwave fluence, is the speed of the highest part of the CME in the coronagraphic images. But the first arrival of an ICME at $1 \mathrm{AU}$ is not necessarily at the position of the Earth. One must therefore characterise which part of the ICME the spacecraft intercepts, the nose or the flank. If it intercepts the flank, we expect that the arrival time estimated from the speed of the top of the CME in the corona is earlier than the first detection of the ICME.

Jian et al. (2006) use the profile of the sum of kinetic and magnetic pressure, $P_{t}$, as a criterion to identify which part of an ICME sweeps over a given spacecraft. They distinguish three groups (their Fig. 5):

G1: The spacecraft encounters the ICME in the vicinity of its centre i.e. near its nose, seeing an increasing $P_{t}$ profile up to a maximum near the centre of the flux rope, and a subsequent decrease.

G3: The spacecraft encounters the border of the ICME, but not the magnetic obstacle; the $P_{t}$ profile is characterised by a rapid rise after the shock, followed by a gradual decay. G2: The spacecraft encounters the ICME on its flank, seeing first an extended sheath region and then the outer regions of the magnetic obstacle: the $P_{t}$ evolution is a mixture of G1 and G3.

We calculated the total pressure profile for all events as the sum of magnetic pressure and plasma thermal pressure:

$$
P_{t}=\frac{B^{2}}{2 \mu_{0}}+\sum_{j} n_{j} k T_{j},
$$

where $\mu_{0}$ is the permeability of the medium and $k$ is the Boltzmann constant. The magnetic field $(B)$ as well as the density $\left(n_{\mathrm{P}}\right)$ and temperature of protons $\left(T_{\mathrm{P}}\right)$ are measured aboard Wind. Plasma pressure is calculated using the abundances for the solar wind of protons $\left(\mathrm{H}^{+}\right)$, $\alpha$ particles $\left(\mathrm{He}^{2+}\right)$ and electrons. We use the parameters of 
(a)

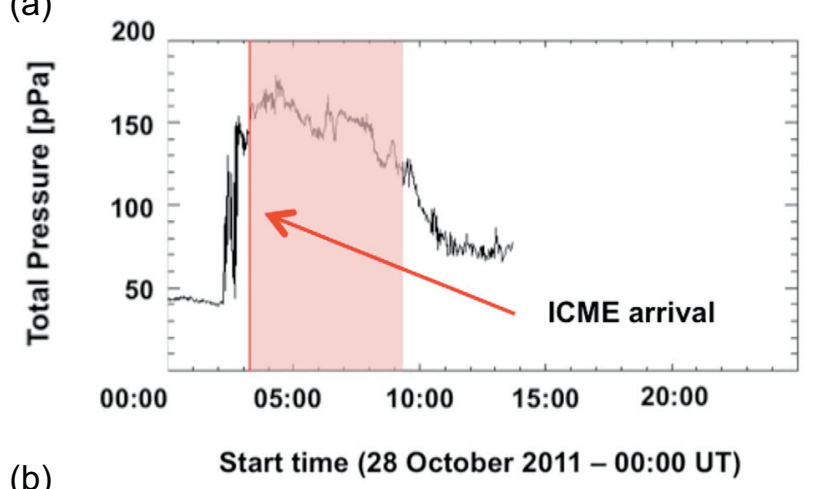

(b)

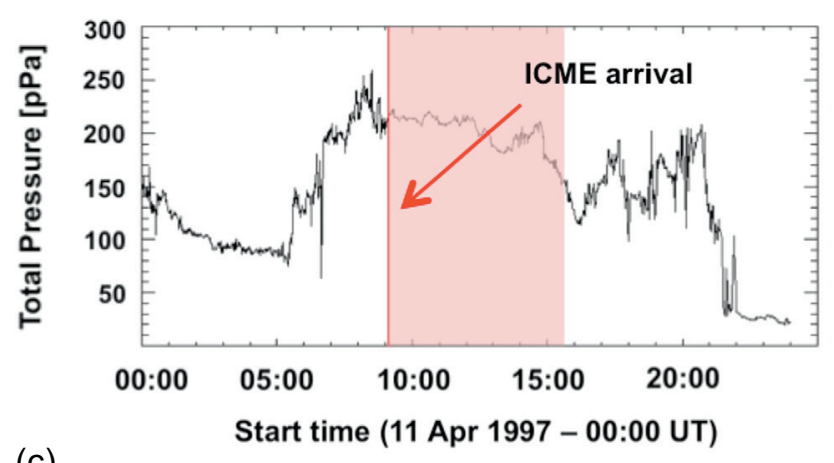

(c)

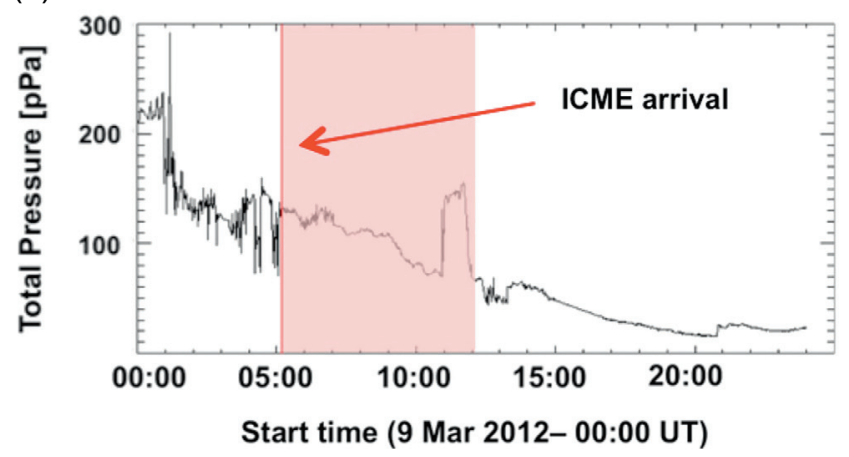

Fig. 6. Examples of total pressure profiles during ICME passages. The red arrow marks the ICME arrival time based on the criterion of temperature drop compared to the expected temperature at the same solar wind speed. The area with a constant low temperature profile is coloured in red. Interpretation of the total pressure profile from the criterion by Jian et al. (2006): (a) G1, spacecraft encounters the ICME near its nose, (b) G2 and (c) G3, spacecraft encounters the ICME flank.

Jian et al. (2006), i.e. the particle densities are such that $95 \%$ are protons, $4 \% \mathrm{He}^{2+}$, the temperature of $\mathrm{He}$ is four times the proton temperature and the electron temperature is $130,000 \mathrm{~K}$. The electron density is taken to be 1.03 times the proton density.

Figure 6 shows examples of total pressure profiles. The vertical red line indicates the ICME arrival based on the drop in the temperature with respect to the expected one for the given solar wind speed. The shaded area represents the region with an almost constant low temperature i.e. the magnetic obstacle of the ICME. Figure 6a shows a well-defined rise of the pressure after the entry into the ICME, with a clear maximum and subsequent somewhat irregular decay. Figure $6 \mathrm{c}$ shows continuous decay of the pressure throughout the passage of the ICME, and Figure $6 \mathrm{~b}$ shows mixed behaviour, without a peak. We found that $4 / 11$ events show the well-defined (a)

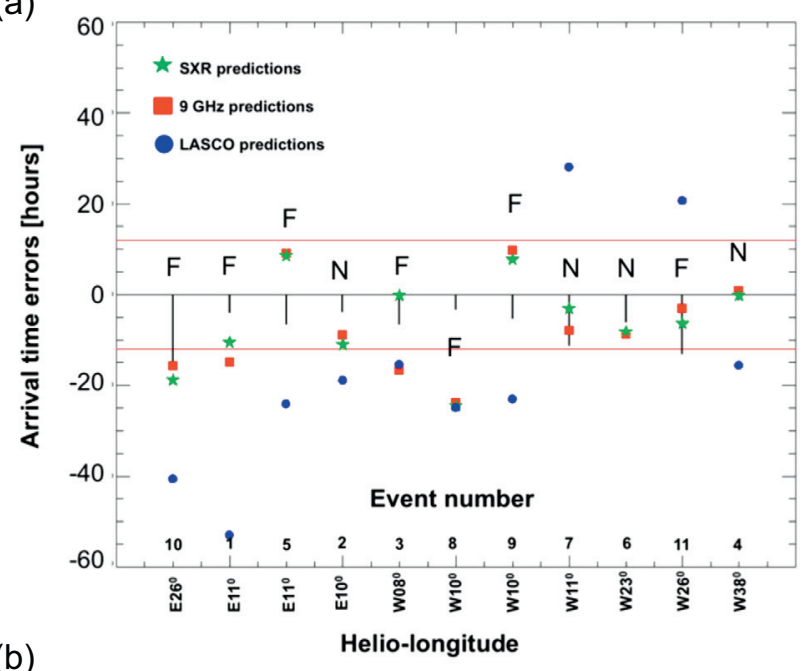

(b)

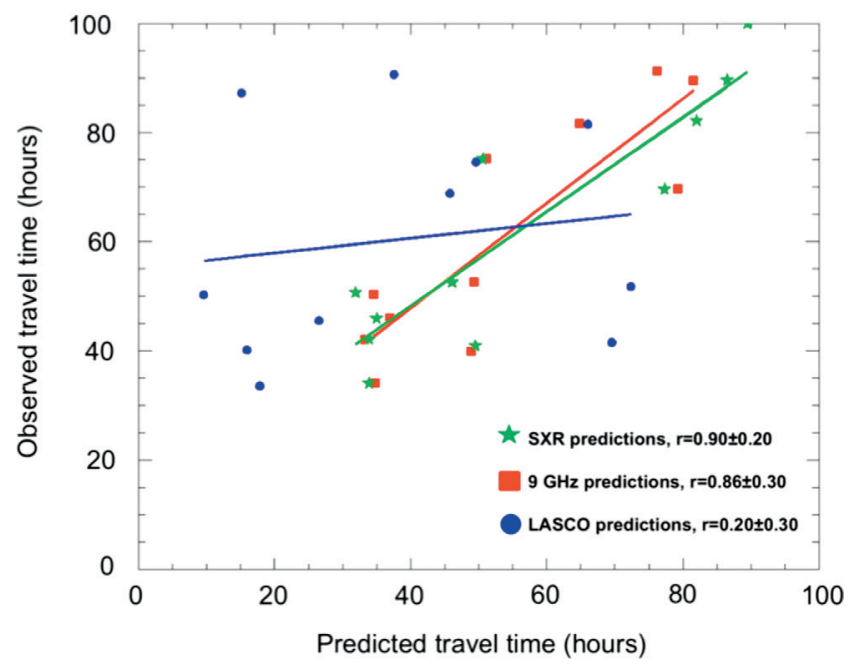

Fig. 7. Errors in arrival time predictions. (a) Travel time errors (predicted - observed arrival times) ordered by increasing longitude. The numbers on the abscissa are the event numbers in Table 3. Green stars: SXR proxy. Red squares: $\phi_{9 \mathrm{GHz}}$. Blue circles: LASCO coronographic observations $\left(V_{\mathrm{EXP}}=2 V_{\mathrm{CAT}}\right)$. The red lines mark the $\pm 12 \mathrm{~h}$ error window while the black vertical lines, the interval between the shock and ICME arrivals. (b) Correlations between observed and predicted travel times.

maximum of total pressure within the ICME. This means that in most cases, the Wind spacecraft intercepted the flank of the ICME.

\subsection{Comparison between predicted and observed arrival times}

The errors of the prediction, that is the difference between predicted and observed arrival times, are shown in Figure 7a. The zero of the ordinate is the observed arrival time of the magnetic driver of the ICME, which has been identified by the drop of the proton temperature. The vertical black lines related to each event show the extent of the sheath region. The labels $\mathrm{F}$ and $\mathrm{N}$ refer to the spacecraft encounter with the ICME: the flank and the nose, respectively. The events are ordered by increasing heliographic longitude of the related flare. Red squares represent the prediction errors using $\phi_{9} \mathrm{GHz}$. For comparison, the prediction errors achieved with the other two methods are also shown: when the coronal speed of the CME is inferred from the SXR fluence (green asterisks) and 
Table 4. Average differences between the predicted and observed ICME arrival times (see Table 3): col. 1 contains the proxy used to infer the CME propagation speed. Cols. 2 and 3: the mean error and the mean absolute error (negative when the predicted arrival precedes the observed ICME arrival) and their standard deviations, respectively. Cols. 4 and 5: the mean error and the mean absolute error and their standard deviations with respect to the sheath arrival.

\begin{tabular}{ccccccc}
\hline \hline Proxies & \multicolumn{2}{c}{ Errors with respect to ICME arrival } & & \multicolumn{2}{c}{ Errors with respect to sheath arrival } \\
\cline { 2 - 3 } & Mean error $[\mathrm{h}]$ & Mean absolute error $[\mathrm{h}]$ & & Mean error $[\mathrm{h}]$ & Mean absolute error $[\mathrm{h}]$ \\
\hline$(1)$ & $(2)$ & $(3)$ & & $(4)$ & $(5)$ \\
Acceleration: equation $(3)$ & & & & \\
Fluence at 9 GHz & $-7.3 \pm 11$ & $-6 \pm 10$ & $9 \pm .8 \pm 7$ & & $8.7 \pm 7$ \\
SXR fluence & $-22 \pm 29$ & $30.6 \pm 18$ & & $8 \pm 6$ \\
Coronographic speed $\left(V_{\mathrm{EXP}}=2 V_{\mathrm{CAT}}\right)$ & $-9 \pm 31$ & $24 \pm 21$ & & $-14.6 \pm 30$ & $28 \pm 17$ \\
Coronographic speed $\left(V_{\mathrm{EXP}}=1.5 V_{\mathrm{CAT}}\right)$ & & & $-2 \pm 33$ & $23 \pm 22$ \\
\hline
\end{tabular}

from coronographic observations of the expansion speed in the plane of the sky ( $\left.V_{\mathrm{EXP}}\right)$ converted to outward propagation speed using the empirical relationship $V_{\mathrm{CME}}=0.88 \mathrm{~V}_{\mathrm{EXP}}$ (blue filled circles, Schwenn et al. 2005). We assume a perfect halo CME where $V_{\text {EXP }}$ is twice the speed reported in the LASCOCME catalogue ${ }^{6}\left(V_{\mathrm{CAT}}\right)$. This probably overestimates the expansion speeds. Therefore, we also quote the errors for the assumption $V_{\mathrm{EXP}}=1.5 V_{\mathrm{CAT}}$. Table 4 contains the mean differences and the mean absolute differences between the predicted and observed arrival times and their standard deviations.

The following conclusions can be drawn from Figure 7 and Table 4:

- The predicted arrival times using the CME speed estimated from SXR and microwave fluences scatter in a broad range between $24 \mathrm{~h}$ before and $10 \mathrm{~h}$ after the observed arrival of the magnetic structure.

- On average, all approximations: the SXR fluence, the $9 \mathrm{GHz}$ fluence and the estimate from the coronographic expansion speed, tend to underestimate the interplanetary travel time of ICMEs.

- The best performance is achieved when the CME speed is derived from the SXR fluence or from $\phi_{9 \mathrm{GHz}}$. The arrival time predictions from SXR fluence and from $9 \mathrm{GHz}$ fluence are usually similar. Both radiative proxies perform better than the coronographic expansion speed. This is confirmed by the high correlations between the observed and predicted arrival times shown in Figure $7 \mathrm{~b}$.

- The arrival time predictions of the four ICMEs, which are intercepted near the nose, range between $12 \mathrm{~h}$ before and one hour after the arrival of the magnetic obstacle. In two of the four cases, the arrival predicted from microwaves or SXR emission occurs during the observed passage of the sheath, in one event it occurs within one hour after the observed arrival of the magnetic obstacle. In the fourth case, the arrival is predicted five hours before the observed arrival of the leading edge of the ICME.

- The largest errors of arrival time prediction from the two proxies are obtained in ICMEs where the flank is intercepted by the spacecraft.

In the previous section, we found that the spacecraft encountered the CME front in events 2, 4, 6 and 7. Although the results in Figure 7 support the simple expectation that the CME arrival time depends on where the spacecraft encounters the ICME, the predictions of events 5, 9 and 11, which are detected on the flanks, are also rather successful.

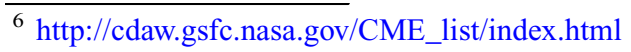

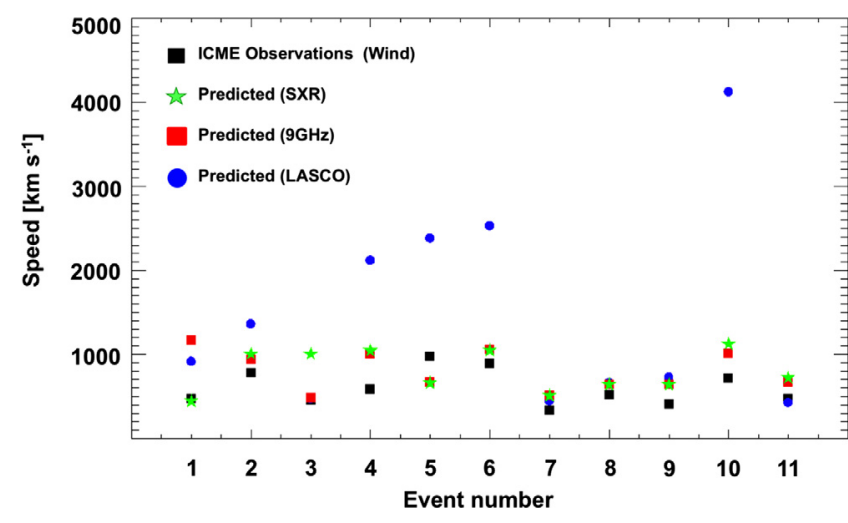

Fig. 8. Comparison between the observed and predicted ICME speeds at $1 \mathrm{AU}$. The black squares represent the speeds measured by Wind. The red filled squares show the predicted speeds from $9 \mathrm{GHz}$ fluence. The green stars mark the predicted speeds from SXR fluence, while the blue circles represent the predicted speeds using coronographic observations. The abscissa shows the event number listed in Table 3.

\subsection{Prediction of ICME speeds at $1 \mathrm{AU}$}

A straightforward further prediction using the CME speed in the corona and the interplanetary transport model is the speed of the ICME near 1 AU. Figure 8 shows the comparison between the ICME speeds predicted at $1 \mathrm{AU}$ and measured at the Wind spacecraft within the ICME, i.e. the speeds determined when the temperature had dropped. We did not find any speed variation that could be related to the expansion of the ICME. Therefore, we consider the measured radial speed as the outward propagation speed of the ICME.

The figure shows that the coronographic proxy predicts in general $(5 / 11)$ the highest ICME speed, well above the observed value. The general overestimation of the ICME speed is in line with the prediction of early arrival times. The microwave fluence predicts speeds much closer to the observations. The mean errors $\Delta V$ and the mean absolute errors $|\Delta V|$ between the predicted and observed speeds are listed in Table 5. The results show comparable errors to the values found by Möstl et al. (2014) $\left(\Delta V=284 \pm 288 \quad\left[\mathrm{~km} \mathrm{~s}^{-1}\right]\right.$ and $\left.|\Delta V|=275 \pm 297\left[\mathrm{~km} \mathrm{~s}^{-1}\right]\right)$ from geometric modelling.

\section{Summary and discussion}

In the present work, an empirical relationship is inferred between the speeds of CMEs at the solar limb, which are supposed to show the outward propagation speeds of the 
CMEs without distortion by projection effects, and the fluence of the associated microwave bursts.

This relationship is then employed to infer the speeds of Earth-directed CMEs, which are fed into a simple analytical model of interplanetary acceleration or deceleration to predict the arrival times and speeds of the ICMEs at 1 AU. The predictions are compared with observations at the Wind spacecraft. Even though our samples are small, they have been carefully characterised such as to avoid spurious associations between limb-CMEs and flares on the one hand, and uncertain ICME arrival times, on the other. Furthermore, we provide a characterisation of the way in which the spacecraft traverses the ICME whose arrival time is determined. This is necessary for comparing the predicted arrival times with observations.

\subsection{Radiative proxies of CME speed in the corona}

The search for microwave counterparts of limb-CMEs was not always successful. Non-thermal microwave signatures may be absent even in some CMEs where an SXR counterpart was clearly identified. This was especially the case in relatively slow CMEs, at speeds below $600 \mathrm{~km} \mathrm{~s}^{-1}$. But even fast CMEs may have no non-thermal microwave signatures. This is the case of CMEs associated with filament eruptions outside active regions. The four CME events listed in Table 1 in Gopalswamy et al. (2015) have speeds between about 950 and $1550 \mathrm{~km} \mathrm{~s}^{-1}$, but have either no detectable microwave burst or a purely thermal one.

When non-thermal microwave bursts are identified, their fluence does show a correlation, with a broad scatter, with the CME speed. Since the sample used to establish the relationship was restricted to CMEs observed near the solar limbs, the scatter can hardly be produced by projection effects on the CME speed measurement. The correlations are similar to what Trottet et al. (2015) found between the CME propagation speed and microwave fluence $(r=0.65 \pm 0.09)$ for events without any restriction in the location of the CME. The scatter must hence be intrinsic to the relationship between the CME speed and the importance of the radiative signatures. It most likely reveals differences from event to event of the energy partitioning between plasma heating, electron acceleration and mass motion. The event dependence of this partitioning was discussed by Reeves \& Moats (2010) in a 2D model of a standard flare-CME scenario, and by Chen \& Kunkel (2010) in a model of flux rope destabilisation.

The empirical relationship can provide an estimation of CME speed in the corona from the SXR or microwave observation. A coronographic observation is needed to decide whether a CME exists or not. Yet, a first prediction of the arrival time and speed of the ICME at $1 \mathrm{AU}$ can be issued from the end of the impulsive microwave burst, when the $\mathrm{CME}$ is only at a few solar radii from the Sun. We have shown that the method has the potential for better prediction than a speed measurement with a coronagraph on the Sun-Earth line.

\subsection{Comparison of radiative proxies with other tools of ICME arrival prediction}

Proxies of CME speeds based on SXR or radio observations have so far not been used in the prediction of ICME arrivals at 1 AU. As shown in Section 3.4, they predict the arrival times of ICMEs at $1 \mathrm{AU}$ with mean absolute errors of 9 to $11 \mathrm{~h}$. The error of the eight best predictions (about $73 \%$ of the
Table 5. ICME speeds at 1 AU: (col. 1) proxy used to infer the ICME speed, (col. 2) mean error value and its standard deviation, and (col. 3) mean absolute error value and its standard deviation.

\begin{tabular}{ccc}
\hline \hline Proxies & \multicolumn{2}{c}{ Errors with respect to ICME } \\
& \multicolumn{2}{c}{ speed } \\
\cline { 2 - 3 } & $\Delta V\left[\mathrm{~km} \mathrm{~s}^{-1}\right]$ & $|\Delta V|\left[\mathrm{km} \mathrm{s}^{-1}\right]$ \\
\hline (1) & $(2)$ & $(3)$ \\
Acceleration: equation (3) & & \\
Fluence at 9 GHz & $196 \pm 243$ & $250 \pm 181$ \\
SXR fluence & $202 \pm 236$ & $264 \pm 155$ \\
Coronographic speed & $870 \pm 1049$ & $878 \pm 1041$ \\
\hline
\end{tabular}

sample of 11 events) is about $\pm 15 \mathrm{~h}$ for the $9 \mathrm{GHz}$ proxy and $\pm 9 \mathrm{~h}$ for the SXR proxy, using the linear model of interplanetary deceleration of Gopalswamy et al. (2001). There is a clear trend to underestimate the ICME travel time, i.e. to predict too early an arrival of the ICME.

While the only relevant observations for a long time were the CME observations in the corona and in situ measurements at $1 \mathrm{AU}$, the advent of heliospheric imaging with the STEREO mission enabled researchers in recent years to track CMEs from the Sun to the Earth (see the early review by Rouillard 2011). Heliospheric imaging was used by Colaninno et al. (2013) to predict the arrival time of nine CMEs, using the graduated cylindrical shell model to remove projection effects. They employed different extrapolations of the height-time trajectory of the CME front beyond a heliocentric distance of $50 \mathrm{R}_{\odot}$ and achieve a prediction of the shock arrival time near $1 \mathrm{AU}$ within $\pm 13 \mathrm{~h}$ for the entire event set, and within $\pm 6 \mathrm{~h}$ for the seven $(78 \%)$ best predictions. Along the same lines, Möstl et al. (2014) extrapolated the height-time trajectories of 22 CME-ICME fronts in heliospheric images. They obtained a prediction error of ICME speeds of about $250-300 \mathrm{~km} \mathrm{~s}^{-1}$, a prediction error of ICME arrival time of 1-7 $\mathrm{h}$ and an absolute prediction error of 7-8 h, depending on the method used. These studies show that heliospheric imaging is an efficient way to track ICMEs through the interplanetary space. It is able to identify non-standard interplanetary acceleration due to interaction of CMEs. Since the prediction relies on the extrapolation beyond some distance from the Sun, about $0.25 \mathrm{AU}$, the advance warning time is reduced to about one day. The Drag-Based Model reveals comparable results. Vršnak et al. (2013) calculated the mean error between the predicted arrival time and observed arrivals of 121 CMEs. They obtain a mean error of about $12 \mathrm{~h}$. Núñez et al. (2016) reported a lower error of about 7-9 $\mathrm{h}$ for the shock arrival time prediction from another empirical dragbased model.

A very sophisticated technique is the combination of nearSun observations with MHD modelling. Millward et al. (2013) utilised the cone model to obtain the CME parameters to be used as input in the WSA-ENLIL+Cone model to forecast the arrivals. They applied their numerical model to a sample of 25 CMEs and found a mean error of around $7.5 \mathrm{~h}$. Similar results are obtained by Vršnak et al. (2014) with the WSAENLIL+Cone model and the analytical drag-based model. The same numerical model was applied by Mays et al. (2015) to a sample of 17 events, where a mean error of about $12 \mathrm{~h}$ was found. Again the prediction errors evaluated by Núñez et al. (2016) were smaller, about 5 h. There is clearly a discrepancy between different evaluations of prediction errors in different publications for the same class of models. 


\subsection{The observational identification of an ICME: a limit to the comparison with predictions}

The evaluation of a successful arrival prediction needs an observational determination of the ICME arrival time at a given spacecraft. This is often ambiguous, depending on the criterion to identify an ICME. Specifically, the time of first detection of the magnetic obstacle within the ICME, which often arrives after a very perturbed shock and sheath region, may be difficult. In the present work, we use the temperature drop with respect to the standard solar wind at comparable speed. It is, however, clear that different criteria commonly used to identify ICME arrivals may on occasion lead to arrival times that differ by several hours. Another problem is the interaction of CMEs, which may significantly alter their interplanetary propagation (e.g., Forbes et al. 2006; Démoulin 2010). Some of the ICMEs in our list are known to undergo interactions (at least events number 8,9 and 10). This may partly explain why different evaluations by similar methods lead to different results in the published literature.

The success of a prediction is also expected to depend on which part of the ICME is intercepted by a spacecraft, the region around the nose or the flank. The mere comparison of predicted ICME arrival times at $1 \mathrm{AU}$ and the observed arrival times at a given spacecraft like Wind is only conclusive if the spacecraft intercepts the nose of the ICME. Most comparisons between predictions and observations suppose tacitly that the nose of the ICME is intercepted. But this is most often not the case: in our sample, 7/11 ICMEs originating within $\pm 40^{\circ}$ from the central meridian were seen on the flanks. The flank of the ICME arrives at the spacecraft when the nose is already beyond $1 \mathrm{AU}$, so that one should indeed expect that many prediction schemes underestimate the travel time. Owens \& Cargill (2004) discuss this effect and show that the arrival at Earth of ICMEs with thick sheath regions is predicted too early. Since the sheath thickness of an ICME with a given speed increases with increasing distance from the nose, they ascribe part of the prediction error to the curvature of the ICME front. These authors use the same set of CME-ICME pairs to determine the interplanetary deceleration and to predict the ICME arrival time. The prediction errors therefore are by construction symmetrically distributed around zero. Our analysis is in remarkable agreement with the expectations, since 9/11 ICME arrivals are predicted about correctly or too early by the SXR and $9 \mathrm{GHz}$ proxies. All predictions that are more than $12 \mathrm{~h}$ ahead of the observed arrival refer to the flanks of ICMEs.

Two alternative interpretations of the early arrival time predictions are the overestimation of the initial CME speed and the uncertainty of the structure whose arrival is predicted, the leading edge or the magnetic obstacle of the ICME. The CME speed is unlikely to be the cause, because the estimates from the microwave fluence tend to be lower than speeds inferred from multi-spacecraft coronographic determinations. The uncertainty of the ICME region may play a role, because the errors in the arrival time prediction are more symmetric with respect to the leading edge of the ICME than with respect to the magnetic obstacle (see Fig. 7). However, the difference between arrival times of the nose and the flank discussed above is inevitable, and must be taken into account when evaluating the predictions. The importance is highlighted by the fact that this difference brings some order into the prediction errors of our test sample.

\subsection{Concluding remarks}

The present work shows that even though many sophisticated numerical and analytical techniques have been developed to forecast ICME arrivals based on coronographic observations, thermal and non-thermal flare-related SXR and microwave emissions are valuable contributions and can provide very early warnings, starting at the time when the CME is first seen in the field of view of a coronagraph on the Sun-Earth line. Although a full validation has not been achieved in the present article, given the small event sample, non-thermal microwave emissions can play a major role, because non-thermal microwave bursts accompany many, though not all, fast CMEs.

The radio observations used in the present work are carried out with rather simple patrol instruments which monitor the whole Sun flux density using parabolic antennae with a typical size of 1 metre. Although the RSTN and Nobeyama patrol instruments do not provide data in real time, there is no technical obstacle to do so. The major issues are a reliable calibration and stable and reliable antenna operations. Because these radio observations are carried out from the ground, they have the additional advantage of being less vulnerable to space weather hazards than space assets.

Acknowledgements. The work presented in this article is based on radio data from the RSTN network (provided through NGDC) and the Nobeyama Radio Polarimeters. The Nobeyama Radio Polarimeters are operated by the Nobeyama Radio Observatory, a branch of National Astronomical Observatory of Japan. Extensive use was made of the CME catalogue, which is generated and maintained at the CDAW Data Center by NASA and The Catholic University of America in cooperation with the Naval Research Laboratory. SOHO is a project of international cooperation between ESA and NASA. Plasma data from the Wind and ACE missions were used through the OMNIweb and CDAWeb servers at NASA GSFC, respectively. Supporting information was provided by the Radio Monitoring website maintained at Paris Observatory with support from CNES. C.S.-M. gratefully acknowledges the financial support of her doctoral studies by the University of Costa Rica and the Ministry of Science, Technology and Telecommunications of Costa Rica (MICITT) through the National Council of Scientific and Technological Research (CONICIT). This research was supported by the Agence Nationale pour la Recherche (ANR/ ASTRID, DGA) project Outils radioastronomiques pour la météorologie de l'espace (ORME, Contract No. ANR-14-ASTR0027) and by the French space agency (CNES). The authors thank P. Démoulin for his help with the identification of ICMEs and many other discussions, A. Isavnin for providing the STEREO solar wind data from the HELCATS project under FP7 EU Contract No. 606692 , and the referees for very constructive comments. The editor thanks the three anonymous referees for their assistance in evaluating this paper.

\section{References}

Bein, B.M., S. Berkebile-Stoiser, A.M. Veronig, M. Temmer, and B. Vršnak. Impulsive acceleration of coronal mass ejections. II. Relation to soft X-ray flares and filament eruptions. Astrophys. $J ., \mathbf{7 5 5}, 44,2012$, DOI: 10.1088/0004-637X/755/1/44.

Cargill, P.J. On the aerodynamic drag force acting on interplanetary coronal mass ejections. Sol. Phys., 221, 135-149, 2004, DOI: 10.1023/B:SOLA.0000033366.10725.a2. 
Caroubalos, C. Contribution à l'étude de l'activité solaire en relation avec ses effects géophysiques. Ann. Astrophys., 27, 333, 1964.

Chen, J., and V. Kunkel. Temporal and physical connection between coronal mass ejections and flares. Astrophys. J., 717, 1105-1122, 2010, DOI: $10.1088 / 0004-637 X / 717 / 2 / 1105$.

Cliver, E.W., J. Feynman, and H.B. Garrett. An estimate of the maximum speed of the solar wind, 1938-1989. J. Geophys. Res., 95, 17103-17112, 1990, DOI: 10.1029/JA095iA10p17103.

Colaninno, R.C., A. Vourlidas, and C.C. Wu. Quantitative comparison of methods for predicting the arrival of coronal mass ejections at Earth based on multiview imaging. J. Geophys. Res. [Space Phys], 118, 6866-6879, 2013, DOI: $10.1002 / 2013 J A 019205$.

Démoulin, P. Interaction of ICMEs with the solar wind. In: M., Maksimovic, K. Issautier, N. Meyer-Vernet, M. Moncuquet, and F. Pantellini, Editors. Twelfth International Solar Wind Conference Vol. 1216 of AIP Conf. Proc., 329-334, 2010, DOI: $10.1063 / 1.3395866$.

Elliott, H.A., D.J. McComas, N.A. Schwadron, J.T. Gosling, R.M. Skoug, G. Gloeckler, and T.H. Zurbuchen. An improved expected temperature formula for identifying interplanetary coronal mass ejections. J. Geophys. Res., 110, A04103, 2005, DOI: 10.1029/2004JA010794.

Forbes, T.G., J.A. Linker, J. Chen, C. Cid, J. Kóta, et al. CME theory and models. Space Sci. Rev., 123, 251-302, 2006, DOI: $10.1007 / \mathrm{s} 11214-006-9019-8$.

Gopalswamy, N. Coronal mass ejections and space weather. In: T., Tsuda, R. Fujii, K. Shibata, and M.A. Geller, Editors. Climate and Weather of the Sun-Earth System (CAWSES) Selected Papers from the 2007 Kyoto Symposium, TERRAPUB, Tokyo, 77-120, 2009.

Gopalswamy, N., A. Lara, R.P. Lepping, M.L. Kaiser, D. Berdichevsky, and O.C. St. Cyr. Interplanetary acceleration of coronal mass ejections. Geophys. Res. Lett., 27, 145-148, 2000, DOI: 10.1029/1999GL003639.

Gopalswamy, N., A. Lara, S. Yashiro, M.L. Kaiser, and R.A. Howard. Predicting the 1-AU arrival times of coronal mass ejections. J. Geophys. Res. [Space Phys], 106, 29207-29218, 2001, DOI: 10.1029/2001JA000177.

Gopalswamy, N., P. Mäkelä, S. Akiyama, S. Yashiro, H. Xie, N. Thakur, and S.W. Kahler. Large solar energetic particle events associated with filament eruptions outside of active regions. Astrophys. J., 806, 8, 2015,

DOI: $10.1088 / 0004-637 \mathrm{X} / 806 / 1 / 8$.

Gopalswamy, N., P. Mäkelä, H. Xie, and S. Yashiro. Testing the empirical shock arrival model using quadrature observations. Space Weather, 11, 661-669, 2013, DOI: 10.1002/2013SW000945.

Gosling, J.T., V. Pizzo, and S.J. Bame. Anomalously low proton temperatures in the solar wind following interplanetary shock waves - evidence for magnetic bottles? J. Geophys. Res. [Space Phys], 78, 2001, 1973, DOI: 10.1029/JA078i013p02001.

Jian, L., C.T. Russell, J.G. Luhmann, and R.M. Skoug. Properties of interplanetary coronal mass ejections at one AU during 1995-2004. Sol. Phys., 239, 393-436, 2006,

DOI: $10.1007 / \mathrm{s} 11207-006-0133-2$.

Maričić, D., B. Vršnak, A.L. Stanger, A.M. Veronig, M. Temmer, and D. Roša. Acceleration phase of coronal mass ejections: II. Synchronization of the energy release in the associated flare. Sol. Phys., 241, 99-112, 2007, DOI: 10.1007/s11207-007-0291-x.

Mays, M.L., A. Taktakishvili, A. Pulkkinen, P.J. MacNeice, L. Rastätter, et al. Ensemble modeling of CMEs using the WSA-ENLIL+Cone model. Sol. Phys., 290, 1775-1814, 2015, DOI: $10.1007 / \mathrm{s} 11207-015-0692-1$.

Millward, G., D. Biesecker, V. Pizzo, and C.A. Koning. An operational software tool for the analysis of coronagraph images: Determining CME parameters for input into the WSA-Enlil heliospheric model. Space Weather, 11, 57-68, 2013, DOI: 10.1002/swe.20024.

Möstl, C., K. Amla, J.R. Hall, P.C. Liewer, E.M. De Jong, et al. Connecting speeds, directions and arrival times of 22 coronal mass ejections from the Sun to 1 AU. Astrophys. J., 787, 119, 2014, DOI: $10.1088 / 0004-637 X / 787 / 2 / 119$.

Nakajima, H., H. Sekiguchi, M. Sawa, K. Kai, and S. Kawashima. The radiometer and polarimeters at 80,35 , and $17 \mathrm{GHz}$ for solar observations at Nobeyama. Publ. Astron. Soc. Jpn., 37, 163-170, 1985.

Núñez, M., T. Nieves-Chinchilla, and A. Pulkkinen. Prediction of shock arrival times from CME and flare data. Space Weather, 2016, in press.

Odstrcil, D., V.J. Pizzo, J.A. Linker, P. Riley, R. Lionello, and Z. Mikic. Initial coupling of coronal and heliospheric numerical magnetohydrodynamic codes. J. Atmos. Sol. Terr. Phys., 66, 1311-1320, 2004, DOI: 10.1016/j.jastp.2004.04.007.

Owens, M., and P. Cargill. Predictions of the arrival time of coronal mass ejections at $1 \mathrm{AU}$ : an analysis of the causes of errors. Ann. Geophys., 22, 661-671, 2004, DOI: 10.5194/angeo-22-661-2004.

Reeves, K.K., and S.J. Moats. Relating coronal mass ejection kinematics and thermal energy release to flare emissions using a model of solar eruptions. Astrophys. J., 712, 429-434, 2010, DOI: $10.1088 / 0004-637 X / 712 / 1 / 429$.

Rouillard, A.P. Relating white light and in situ observations of coronal mass ejections: a review. J. Atmos. Sol. Terr. Phys., 73 , 1201-1213, 2011, DOI: 10.1016/j.jastp.2010.08.015.

Salas-Matamoros, C., and K.-L. Klein. On the statistical relationship between CME speed and soft X-ray flux and fluence of the associated flare. Sol. Phys., 290, 1337-1353, 2015, DOI: $10.1007 / \mathrm{s} 11207-015-0677-0$.

Schwenn, R., A. Dal Lago, E. Huttunen, and W.D. Gonzalez. The association of coronal mass ejections with their effects near the Earth. Ann. Geophys., 23, 1033-1059, 2005, DOI: 10.5194/angeo-23-1033-2005.

Shi, T., Y. Wang, L. Wan, X. Cheng, M. Ding, and J. Zhang. Predicting the arrival time of coronal mass ejections with the graduated cylindrical shell and drag force model. Astrophys. J., 806, 271, 2015, DOI: 10.1088/0004-637X/806/2/271.

Temmer, M., A.M. Veronig, V. Peinhart, and B. Vršnak. Asymmetry in the CME-CME interaction process for the events from 2011 February 14-15. Astrophys. J., 785, 85, 2014, DOI: $10.1088 / 0004-637 \mathrm{X} / 785 / 2 / 85$

Thernisien, A., A. Vourlidas, and R.A. Howard. Forward modeling of coronal mass ejections using STEREO/SECCHI data. Sol. Phys., 256, 111-130, 2009, DOI: $10.1007 / \mathrm{s} 11207-009-9346-5$.

Tobiska, W.K., D. Knipp, W.J. Burke, D. Bouwer, J. Bailey, D. Odstrcil, M.P. Hagan, J. Gannon, and B.R. Bowman. The Anemomilos prediction methodology for Dst. Space Weather, 11, 490-508, 2013, DOI: 10.1002/swe. 20094.

Trottet, G., S. Samwel, K.-L. Klein, T. Dudok deWit, and R. Miteva. Statistical evidence for contributions of flares and coronal mass ejections to major solar energetic particle events. Sol. Phys., 290, 819-839, 2015, DOI: 10.1007/s11207-014-0628-1.

Vršnak, B., D. Ruždjak, D. Sudar, and N. Gopalswamy. Kinematics of coronal mass ejections between 2 and 30 solar radii. What can be learned about forces governing the eruption? $A \& A, \mathbf{4 2 3}$, 717-728, 2004, DOI: 10.1051/0004-6361:20047169.

Vršnak, B., M. Temmer, T. Žic, A. Taktakishvili, M. Dumbović, C. Möstl, A.M. Veronig, M.L. Mays, and D. Odstrčil. Heliospheric propagation of coronal mass ejections: comparison of numerical WSA ENLIL cone model and analytical drag-based model. Astrophys. J. Suppl., 213, 21, 2014,

DOI: $10.1088 / 0067-0049 / 213 / 2 / 21$.

Vršnak, B., and T. Žic. Transit times of interplanetary coronal mass ejections and the solar wind speed. $A \& A, 472,937-943,2007$, DOI: 10.1051/0004-6361:20077499. 
Vršnak, B., T. Žic, D. Vrbanec, M. Temmer, T. Rollett, et al. Propagation of interplanetary coronal mass ejections: the dragbased model. Sol. Phys., 285, 295-315, 2013, DOI: $10.1007 / \mathrm{s} 11207-012-0035-4$

Wu, C.-C., M. Dryer, S.T. Wu, B.E. Wood, C.D. Fry, K. Liou, and S. Plunkett. Global three-dimensional simulation of the interplanetary evolution of the observed geoeffective coronal mass ejection during the epoch 1-4 August 2010. J. Geophys. Res. [Space Phys], 116, A12103, 2011, DOI: 10.1029/2011JA016947.

Zhang, J., I.G. Richardson, D.F. Webb, N. Gopalswamy, and E. Huttunen. Solar and interplanetary sources of major geomagnetic storms (Dst $=-100 \mathrm{nT}$ ) during 1996-2005. J. Geophys. Res., 112, 10102, 2007, DOI: 10.1029/2007JA012321.

Cite this article as: Matamoros CS, Klein KL \& Trottet G. Microwave radio emissions as a proxy for coronal mass ejection speed in arrival predictions of interplanetary coronal mass ejections at 1 AU. J. Space Weather Space Clim., 7, A2, 2017, DOI: 10.1051/swsc/2016038. 